\title{
Purumllaqta de Cuimal y el área de San Jerónimo (Amazonas) durante la hegemonía inca
}

Recibido: $17 / 05 / 19$

Aprobado: 04/08/19

\author{
Anthony Villar Quintana' \\ Universidad Nacional Mayor de San Marcos \\ anlex1234@gmail.com
}

\begin{abstract}
RESUMEN
El presente artículo aborda el tema de la hegemonía inca en el área de la actual comunidad campesina de San Jerónimo, en la margen izquierda del rio Utcubamba, Amazonas. Considerando principalmente al sitio de Purumllaqta de Cuimal y su relación con las estructuras funerarias de cerro El Tigre y los sitios con arte rupestre de Suinte, Ayasamana y Chumoc, aproximándonos así a la importancia de esta área para el Tahuantinsuyo.
\end{abstract}

Palabras clave: Purumllaqta de Cuimal, hegemonía inca, Amazonas.

\section{Purumllaqta de Cuimal and the area of San Jerónimo (Amazonas) during the Inca hegemony}

\begin{abstract}
This article addresses the issue of Inca hegemony in the area of the current community of San Jerónimo, on the left bank of the Utcubamba River, Amazonas. Considering mainly the site of Purumllaqta de Cuimal and its relationship with the funerary structures of Cerro El Tigre and the rock art sites of Suinte, Ayasamana and Chumoc, we approach the importance of this area for the Tahuantinsuyo.
\end{abstract}

KeYwords: Purumllaqta de Cuimal, Inca hegemony, Amazonas.

Bachiller en Ciencias Sociales, especialidad Arqueología, interesado en investigaciones sobre el Periodo Intermedio Tardío y Horizonte Tardío, especialmente en el área amazónica andina. 


\section{Introducción}

A mazonas constituyó el escenario de distintas sociedades a través del tiempo, una de estas la constituyeron los enigmáticos pobladores que modificaron el paisaje natural construyendo distintos asentamientos con edificios de planta circular, contando además con diversas prácticas funerarias. Estas sociedades fueron conquistadas por el imperio incaico durante su periodo expansivo, y ulteriormente por los españoles, quienes modificaron el sistema social anterior.

Existen muchos materiales que muestran la influencia inca en los diversos asentamientos y espacios del territorio amazonense, ya sea en arquitectura, cerámica, metales, arte rupestre, u otros. Sin embargo muchos de estos aún no han sido abordados por la investigación arqueológica, a pesar del creciente interés en el área de diversos investigadores. Por lo cual tratamos de contribuir al tema mediante nuestras apreciaciones referentes a Purumllaqta de Cuimal y otros sitios en sus inmediaciones, dentro del área perteneciente a la comunidad de San Jerónimo, en la margen izquierda del río Utcubamba, buscando aproximarnos a la importancia de dicha área durante la hegemonía inca.

Teniendo en cuenta, la presencia material de filiación inca en asentamientos próximos, como los referentes al complejo arqueológico de Kuélap (Ruiz Estrada 2009 [1972]: 15) en la provincia de Luya y los del área chachapoyana (Schjellerup 2005; Ruiz Estrada 2004; Crandall 2017). Asimismo no debemos olvidar la necesidad del imperio incaico de anexar todos los territorios del actual departamento de Amazonas y así proseguir con sus conquistas hacia el norte, en los actuales países de Ecuador y Colombia.

En el presente trabajo consignamos los datos obtenidos en muchas de nuestras visitas al territorio de San Jerónimo, acompañados de Miuler Villar Sánchez, quien lleva recorriendo el área por más de dos décadas, ubicando así distintos sitios arqueológicos, muchas veces por referencia de los lugareños. Siendo el principal motivo que nos llevó a escribir el presente artículo, las consideraciones del sitio de Purumllaqta de Cuimal como el asentamiento que habitaron los comuneros de San Jerónimo antes de trasladarse a su actual poblado.

\section{Ubicación y características geográficas}

Purumllaqta de Cuimal se ubica en la parte llana de un cerro, en la Cordillera Oriental de los Andes, entre las quebradas Gapacio (al norte) y Clamal (al sur), ambos afluentes del rio Utcubamba por su margen izquierda, en la cuenca media de este. Políticamente pertenece al territorio de la actual comunidad campesina de San Jerónimo (Fig. 1), anexo del distrito de Jazán, provincia de Bongará, región Amazonas; sin embargo hace menos de un siglo la comunidad perteneció al distrito de San Jerónimo de Paclas, en la provincia de Luya. De acuerdo a las coordenadas UTM, según el sistema WGS 84, el sitio se ubica en el cuadrante 17M, 0830511 E y 9335833 N, a 2516 m.s.n.m.

La ecorregión en la que se ubica Purumllaqta de Cuimal corresponde a la Ceja de Selva, también denominada como Amazonia Andina (Morales 1998: 621), la cual presenta una diversidad de climas y paisajes de Bosque Pluvial Montano Bajo Tropical, con ambientes cubiertos por un tupido bosque tropical oculto muchas veces por neblinas (Kauffmann \& Ligabue 2003: 27). Como gran parte de la ecorregión, el área presenta una topográfica irregular, con quebradas profundas, altos acantilados y majestuosos cerros, destacando entre estos últimos el cerro Gololque, ya que constituiría un Apu con atributos sobre el agua, teniendo el poder de hacer $1 \operatorname{lover}^{2} \mathrm{y}$ portar recursos auríferos (Véase: Torres 1986: 7-8; 1994: 19-20; Taylor 1996: 65-67).

\section{Investigaciones arqueológicas en San Jerónimo}

El territorio perteneciente a la comunidad de San Jerónimo, es conocido arqueológicamente por la presencia de purunmachu(s) o sarcófagos antropomorfos, en un acantilado del cerro denominado El Tigre. Si bien pueden existir muchos purunmachu(s) en este acantilado y en otros circundantes ${ }^{3}$, hasta el mo-

2 Los comuneros actuales de San Jerónimo consideran que si visitan Gololque o realizan quemas en este o en los cerros cercanos sin duda lloverá, incluso torrencialmente.

3 Estos son conocidos por algunos lugareños, sin embargo hasta el momento no se ha realizado un inventario por medio de prospecciones arqueológicas. 


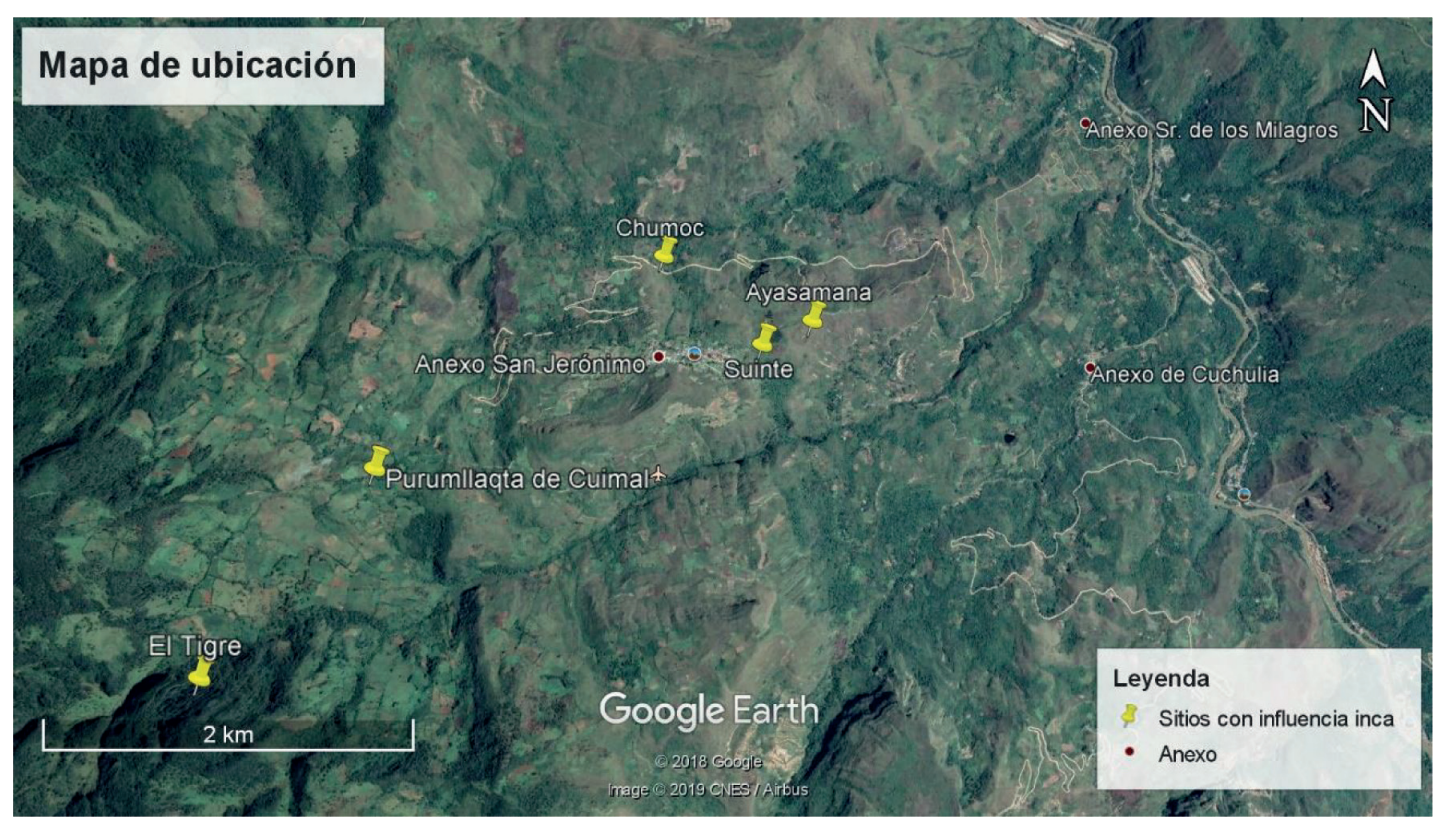

Fig.1. Mapa de ubicación de Purumllaqta de Cuimal y otros sitios arqueológicos mencionados en la presente investigación, elaborado mediante Google Earth.

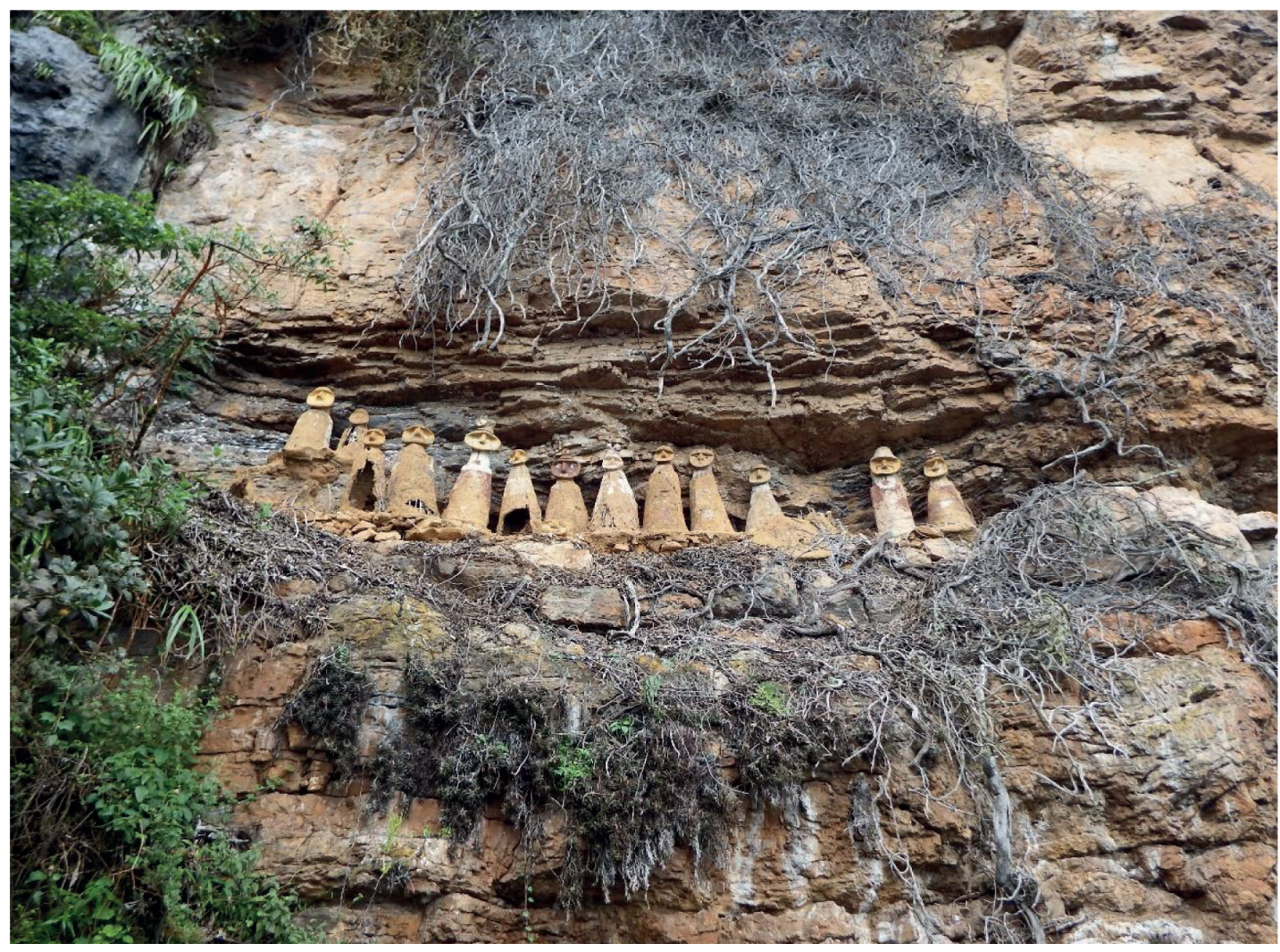

Fig.2. Segundo grupo de purunmachu(s) reportados en cerro El Tigre.

mento se han reportado la existencia de tres grupos de estos, cada uno en un abrigo rocoso.

El primer grupo fue localizado por el arqueólogo Newman Aguilar y el profesor Miuler Villar en 1998, dándose a conocer a los medios en 2011 (Quintana 2011; Villar 2012). El segundo grupo de purunmachu(s) (Fig.2), a 400 metros del primero, fue hallado posteriormente en 2013 por un grupo de jóvenes conducidos por Gruver Chávez (Quintana 2013; Villar 2013a); el mismo año fue localizado el tercer grupo por el profesor Miuler Villar, sumando entre los tres grupos más de cua- 

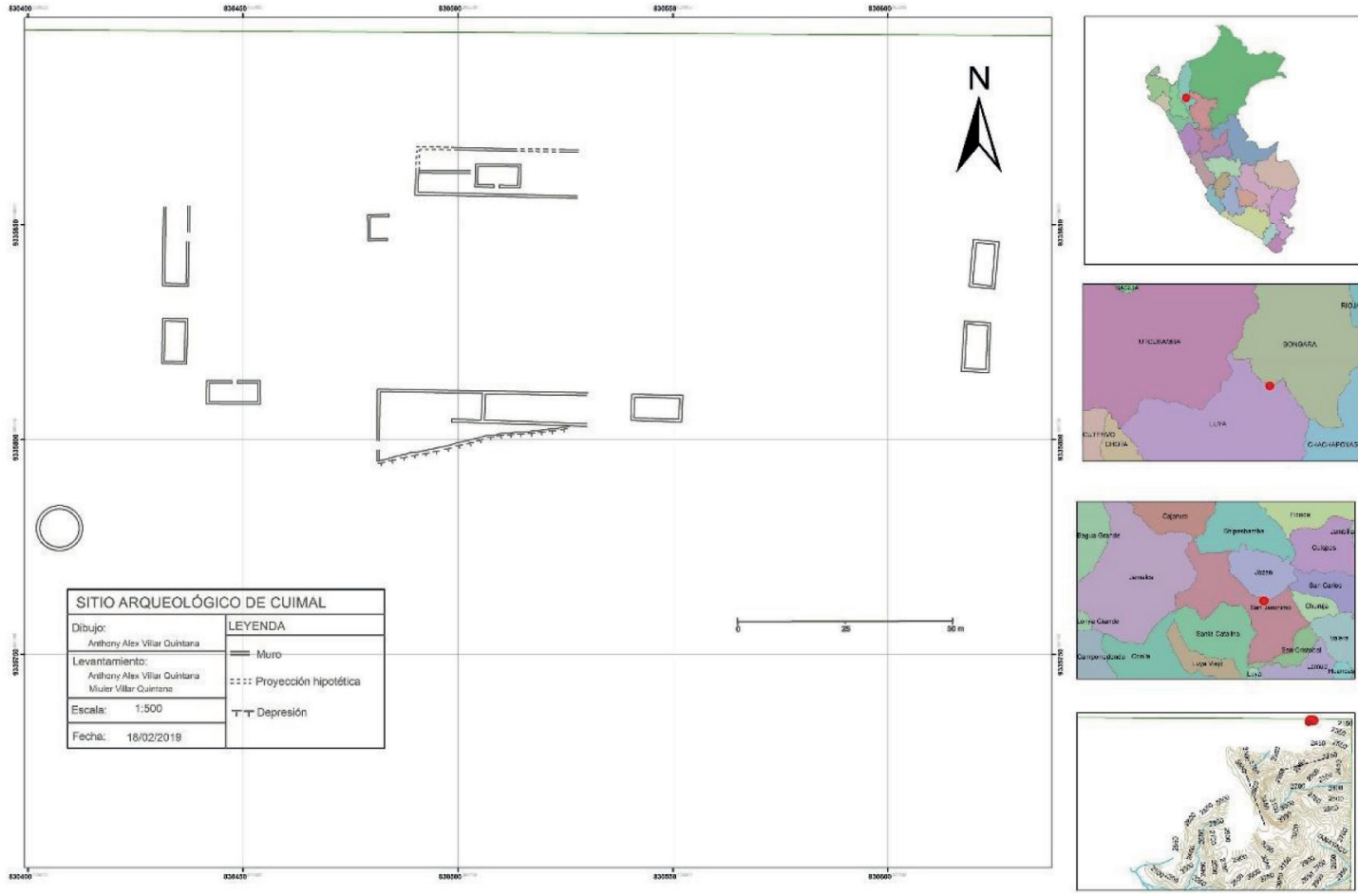

Fig.3

Plano de

Purumllaqta de Cuimal.

tro decenas de purunmachu(s) en el acantilado (Villar 2013b).

El mismo año integrantes del Centro Mallqui y Espeleo Kandil, encargados por la arqueóloga Sonia Guillén, realizaron un registro del segundo grupo de purunmachu(s), identificando además ciertas características de un posible grupo de edificios funerarios en un abrigo próximo al sitio (Centro Mallqui \& Espeleo Kandil 2013). Siendo también este grupo visitado posteriormente por el arqueólogo Federico Kauffmann, quien realizo gráficos y algunas apreciaciones de los mismos (Kauffmann 2017: 171-177).

Los recursos arqueológicos de San Jerónimo no solo se limitan a purunmachu(s) o sarcófagos antropomorfos, ya que en su territorio también son observables otras expresiones culturales prehispánicas, como asentamientos de diversas complejidades, estructuras funerarias en acantilados y arte rupestre; sobre esta última tenemos referencias de Petter Arana y Horacio Zuta sobre representaciones de figuras concéntricas, circulares y escalonadas pintadas en abrigos rocosos de San Jerónimo (Arana \& Zuta 2009: 52).

\section{Cuimal en las fuentes etnohistóricas y orales}

Existen documentos de finales del siglo xvi, como el escrito por Francisco Grandez de Salinas en $1598^{4}$ donde hace referencia al pueblo de San Gerónimo de Cuimal y su territorio dentro de las provincias de Luya y Chillaos, así como a su cacique Juán Llanca (Grandez 1598). Durante la segunda mitad del siglo xviII, tanto el médico y cosmógrafo Francisco Antonio Cosme Bueno y Alegre (1764: 32) como el geógrafo e historiador Antonio de Alcedo (1786: 727), mencionan al pueblo de San Gerónimo de Cunumal, como uno de los dos anexos ${ }^{5}$ del curato de Olto, dentro de la Provincia y Corregimiento de Luya y Chillaos.

En la tradición oral actual, de la comunidad de San Jerónimo, se hace referencia a Cuimal como «Purumllaqta» o "pueblo viejo»; según una leyenda dicho asentamiento habría sido abandonada debido a que muchos niños desaparecían al seguir a un ave roja, que salía de una fuente de agua (Torres 1986: 8; 1994: 16; UGEL Bongará 2006: 192-193), por lo cual sus pobladores decidieron trasladarse a

4 La comunidad de San Jerónimo conserva el manuscrito y una trascripción de esta, realizada por el historiador y paleógrafo Raúl Rivera Serna.

5 El pueblo de Chosgón correspondió al otro anexo del curato de Olto. 


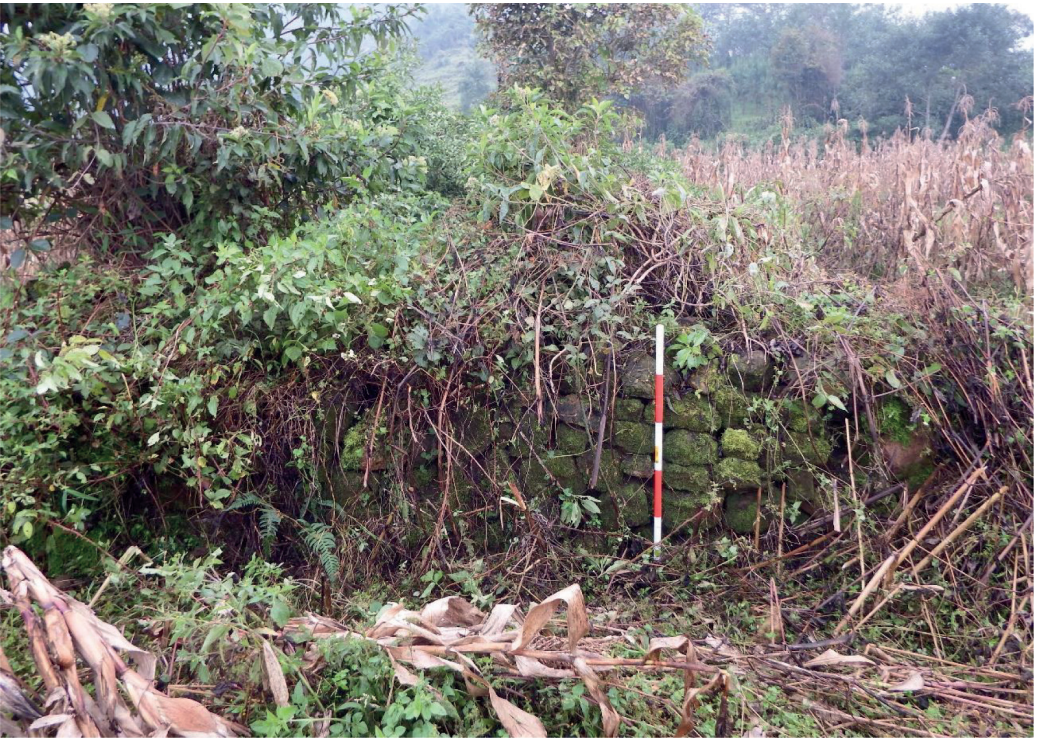

Fig.4. Muro de pirca en uno de los edificios de Purumllaqta de Cuimal.

Sicsicpata (UGEL Bongará 2006: 193), este último correspondería al antiguo nombre del pueblo actual de San Jerónimo.

\section{Descripción y análisis del asentamiento}

Purumllaqta de Cuimal se encuentra en un área llana, presentando mal estado de conservación, debido al uso intensivo del área en actividades agropecuarias, lo cual hace difícil identificar los diversos elementos arquitectónicos y distinguir sus periodos de construcción. Aun así, reconocimos la existencia de edificios (Fig. 3) construidos mediante pirca tanto de planta ortogonal como circular, de este último observamos solo una estructura, la cual se encuentra en el suroeste del sitio, con un diámetro de 10. 70 metros. Este edificio de planta circular demuestra la influencia local dentro del asentamiento de Purumllaqta de Cuimal, ya que esta forma se encuentra difundida por todo el territorio perteneciente a las sociedades "Chachapoya» ${ }^{6}$.

La parte central del asentamiento corresponde a una plaza ortogonal, definida por edificios ortogonales, la cual se encuentra orientada siguiendo los puntos cardinales. Debido al mal estado de conservación del sitio solo se conoce con exactitud una de

6 Término usado para referirse al conjunto de sociedades que habitaron el territorio (v.g. Chachapoya, Chilcho, Luya y Chillao), posteriormente anexadas al imperio incaico. las dimensiones de dicha plaza, la cual contaría con 45 metros de sur a norte y más de 50 metros de oeste a este. Cabe resaltar la presencia de un edificio de planta cuadrangular en el noroeste de la plaza, probablemente construido en periodos post-colombinos ya que se encontraría dentro de la plaza.

$\mathrm{Al}$ este de la plaza se ubican tres edificios de planta rectangular formando una "L», dos orientados de sur a norte y ubicados consecutivamente, y un tercero orientado de oeste a este, ubicado al sureste de estos. Cabe resaltar que es el tercer edificio descrito el que se encuentra más conservado, observándose en este la tecnología constructiva de los edificios en Purumllaqta de Cuimal, empleándose piedras cuadrangulares de regular tamaño para formar las paredes mediante pircas (Fig. 4).

Como se mencionó anteriormente, el estado de conservación impide observar muchas características de los edificios, incluyendo la configuración arquitectónica general. Sin embargo notamos que los tres edificios tendrían sus respectivos accesos orientados hacia un espacio común, lo cual indicaría que estas estructuras junto a otras, ya destruidas, habrían conformado una kancha, un patrón típico incaico.

Hacia el sur de la plaza se ubica una estructura de planta rectangular alargada orientada de oeste a este, con 7.70 metro de ancho y más de 23 metros de largo, cuyo acceso o accesos habrían estado orientadas hacia la plaza. Al oeste de este edificio se ubica un posible patio en su lado oeste, definido por muros de pirca, teniendo un recinto en el interior de este espacio, conformado por las paredes sur y norte del edificio alargado, con el cual comparte una pared divisoria, posiblemente adosada a dicha estructura para crear así dos espacios. Por otra parte a 10 metros al este del edificio ortogonal alargado se ubica un estructura de planta cuadrangular, orientada de oeste a este, con 11.70 metros de largo por 6 de ancho.

$\mathrm{Al}$ este de la plaza se distinguen dos edificios de planta rectangular orientados de sur a norte, cuyos vanos de acceso se encontrarían en su lado oeste. Ambos edificios cuentan con dimensiones de aproximadamente 11 metros de largo por 6 de ancho. Cabe resaltar que inmediatamente al este de estos edificios queda un gran espacio sin estructuras visibles, creemos que habría presentado una disposición similar a la observada en los edificios que definían en lado opuesto de la plaza, conformando una posible kancha. 
Definiendo el lado norte de la plaza se ubica una gran estructura de planta rectangular alargada con una orientación de oeste a este, de aproximadamente 11.20 metros de ancho por más de 38 metros de largo. Esta estructura sirvió de plataforma para la construcción de un edifico de planta rectangular con la misma orientación, con 3.50 metros de ancho por 10.40 de largo, y de un posible patio contiguo al oeste de dicho edificio.

Debido a sus características, es muy probable que la estructura rectangular alargada ubicada al lado sur de la plaza habría constituido una kallanka durante el Horizonte Tardío, idea respaldada además por su ubicación en una ladera empinada al norte, lo cual indicaría que el acceso hacia el edificio se realizaba desde la plaza, siendo esta una de las características básicas de la kallanka. Siendo esta estructura modificada en periodos posteriores al construirse un patio y un recinto sobre la esta.

Es así que la planimetría del sitio, evidencia la existencia de un asentamiento durante el Horizonte Tardío, compuesto por una plaza ortogonal definida por edificios con formas típicas incaicas como kanchas y kallankas, las cuales habrían sido modificadas durante el periodo colonial temprano y/o posteriormente. Cabe resaltar que en Cuimal el señor Ángel Ventura Ocampo, uno de los pobladores de San Jerónimo, halló un hacha metálica de estilo inca (Fig. 5), lo cual prueba que parte del asentamiento habría sido construido durante el Horizonte Tardío; cabe resaltar que objetos similares fueron hallados en Pachacamac (Vetter 2008: 193, 200), Purumllaqta de Cheto (Ruiz Barcellos 2001 citado por Crandall 2017: 286) y Cochabamba (Schjellerup 2005: 254), los últimos dos correspondientes a centros administrativos inca en el área amazonense.

\section{Otros sitios con influencia inca en territorio de San Jerónimo}

Alrededor de Purumllaqta de Cuimal, dentro del territorio de San Jerónimo, observamos otros sitios que presentarían materiales arqueológicos de filiación y/o influencia inca, como:

El Tigre: Los acantilados de cerro El Tigre fueron soportes de múltiples contextos funerarios, de estos nos llamó la atención dos fotografías captura-

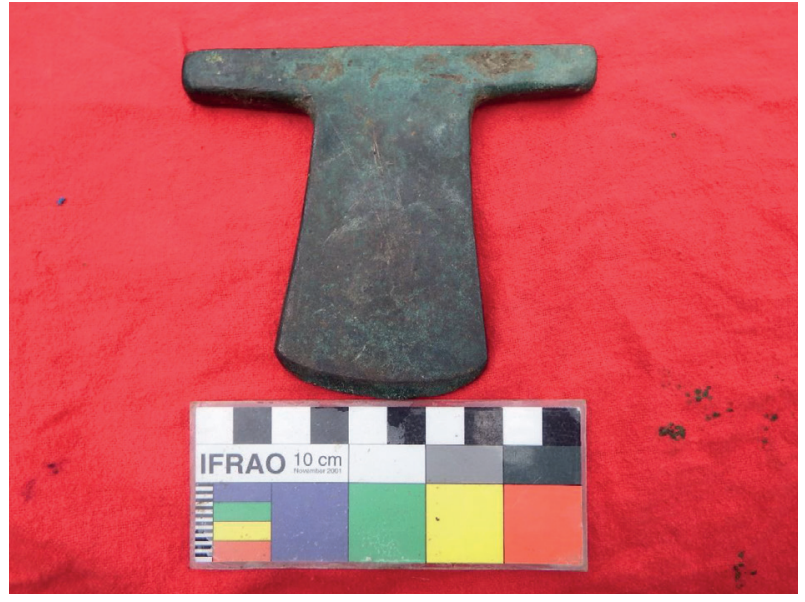

Fig.5. Hacha metálica de estilo inca, hallada en Purumllaqta de Cuimal.

das por el fotógrafo Martin Chumbe y conservadas en la Institución Educativa 18186 «Tito y Sofia»-San Jerónimo, estas corresponden a unos fardos funerarios (Fig. 6) encontrados en las edificaciones de piedra y barro ubicados en uno de los abrigos ${ }^{7}$.

Lamentablemente dichos fardos han desaparecido por obra de saqueadores, por lo cual estas fotografías constituyen una fuente de información, permitiéndonos observar dos cuerpos humanos momificados, uno de los cuales se encontraba en mejor estado de conservación, permitiéndonos observar más detalles del proceso de enfardelamiento, el cual habría constituido en colocar los cuerpos en posición de cuclillas con las manos en el rostro ${ }^{8}$, para ser envueltos con mantos, sujetados por una especie de red hecha de material vegetal.

Según Sonia Guillén (2002: 359-361; 2003: 297-298) fueron los incas quienes introdujeron la momificación en las costumbres funerarias de las sociedades locales en Amazonas, ya que antes de ser conquistados habrían realizado entierros secundarios. Por lo cual consideramos que estas dos momias habrían sido depositadas en el sitio durante la hegemonía incaica, siendo trasladadas al lugar desde asentamientos próximos como Purumllaqta de Cuimal, Pińaurco, Cashco u otros más en el cerro Gololque y en el mismo cerro El Tigre, los cuales presentan arquitectura de estilo arquitectónico local.

7 Se trata del mismo abrigo con estructuras funerarias observadas y mencionadas anteriormente por otros investigadores (Centro Mallqui \& Espeleokandil 2013: 2-6; Kauffmann 2017: 173)

8 No sabemos si el proceso de momificación fue artificial o natural, ya que requeriría de un análisis más detallado, lo cual será imposible realizar ahora que las momias se encuentran desaparecidas. 


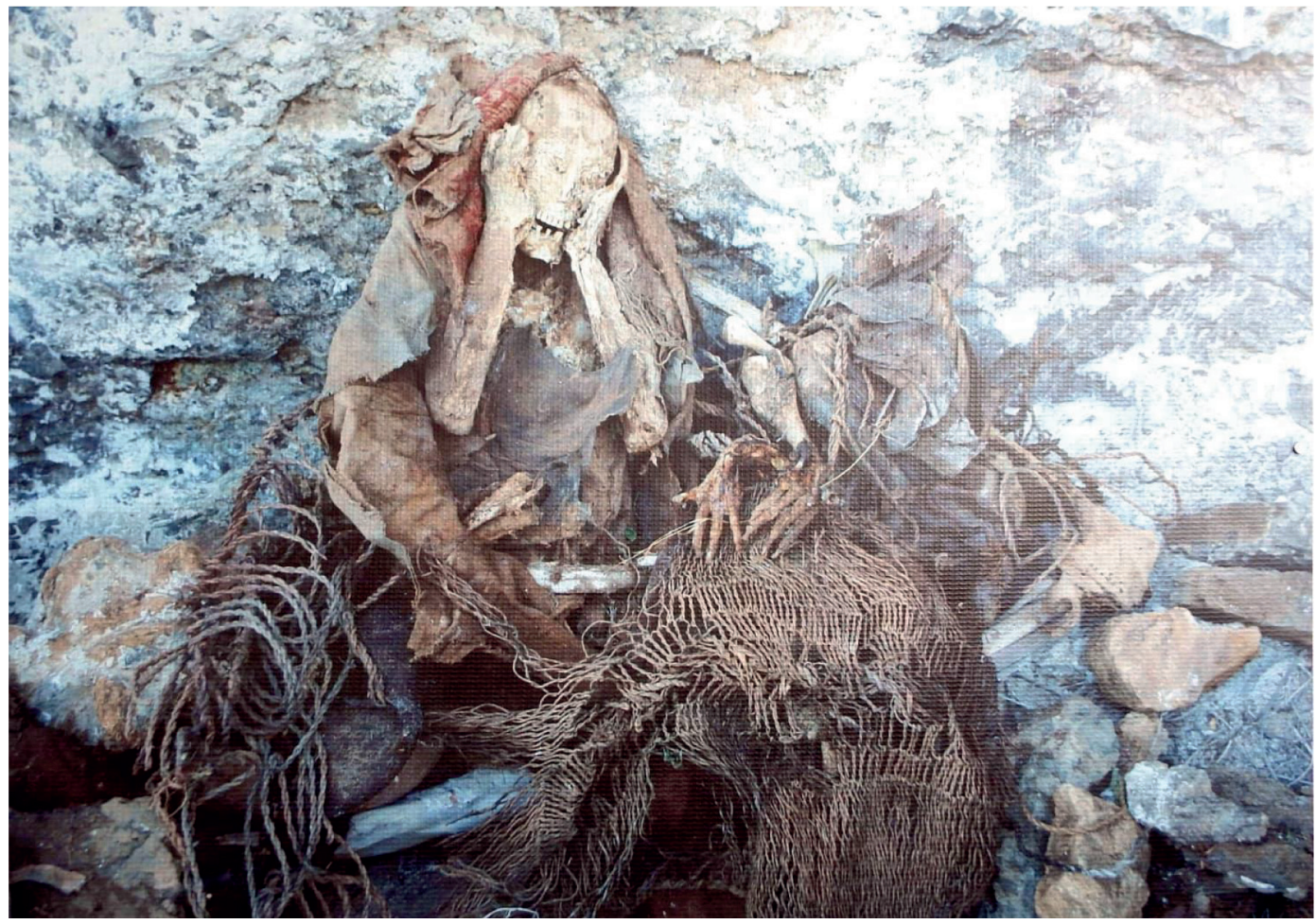

Fig.6. Fardos

funerarios

encontrados en

las edificaciones

de piedra y

barro ubicados

en uno de los

abrigos rocosos

de cerro El

Tigre. Fotografía

capturada por

Martín Chumbe.

Suinte: Ubicado inmediatamente al este de la comunidad de San Jerónimo, cerca al sector denominado la escalera en el camino de herradura que conduce hasta las orillas del río Utcubamba. Situado en un abrigo rocoso, a 2095 m.s.n.m., donde se plasmaron diversos motivos rupestres antropomorfos, zoomor- fos y geométricos en color rojo, destacando entre estos la representación de una túnica andina (unku), de 20 por 35 centímetros, con una pechera en " $\mathrm{V}$ » escalonada (Fig. 7).

La representación de unkus en el arte rupestre, se habría difundido por gran parte del Tahuantinsuyo

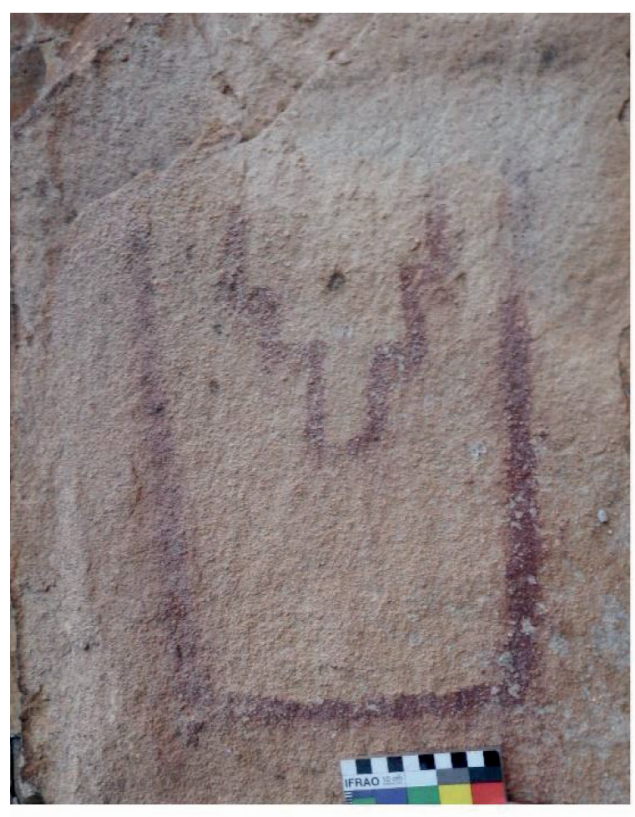

(1)

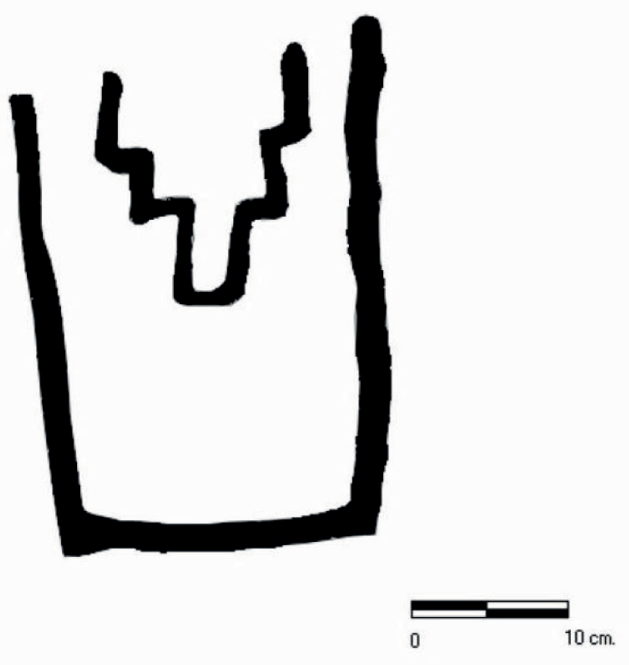

(2)
Fig. 7. Pintura rupestre, representando una túnica andina (unku) con pechera en «V» escalonada, en Suinte. Fotografía (1) y calco (2). 


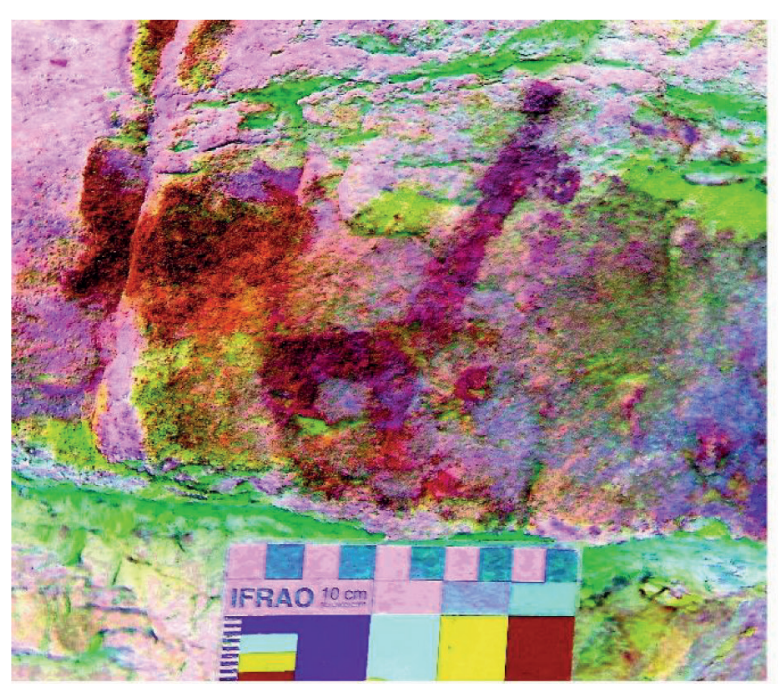

(1)

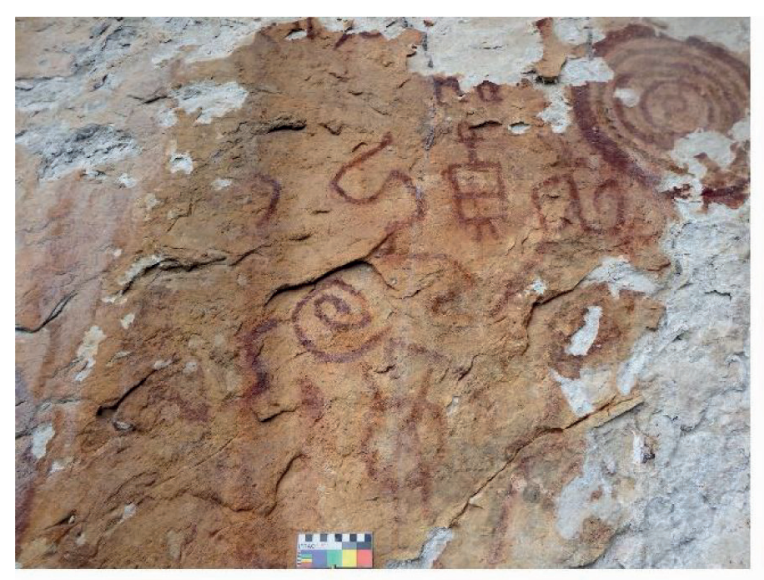

(1)

(Hostnig 2006; 2008; 2017; Sepúlveda 2004; Ruiz 2002; Ruiz Estrada 2007; Ruiz \& Chorolque 2012; Berenguer 2013), siendo además el representado en Suinte un tipo muy resaltante en el arte textil inca, llegando a confeccionarse tanto en grandes tamańos como en miniaturas, estas últimas como parte de diversas ofrendas, correspondientes a personajes antropomorfos metálicos de pequeño tamaño.

Ayasamana: Ubicado al este de la comunidad de San Jerónimo, a casi medio kilómetro de Suinte. Situado en un abrigo rocoso, a una altura aproximada de 1932 m.s.n.m., donde se plasmaron diversas representaciones en pinturas rupestres correspondientes a motivos antropomorfos, zoomorfos y geométricos en color rojo, destacando la representación de un camélido dispuesto de perfil, con la cola recta, la oreja erguida y posibles autopodios (pies) en sus ex-

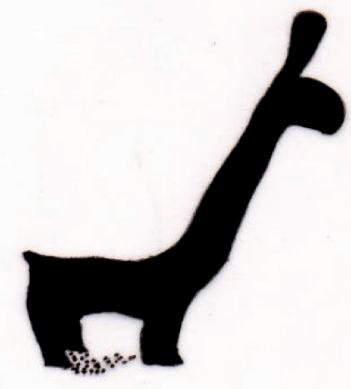

Fig. 8. Camélido de estilo inca representado en el arte rupestre de Ayasamana. Fotografía procesada en software D-Stretch, canal «crgb»(1) y calco de la representación (2).

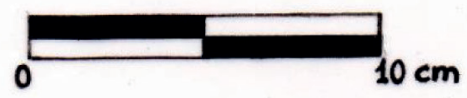

(2)

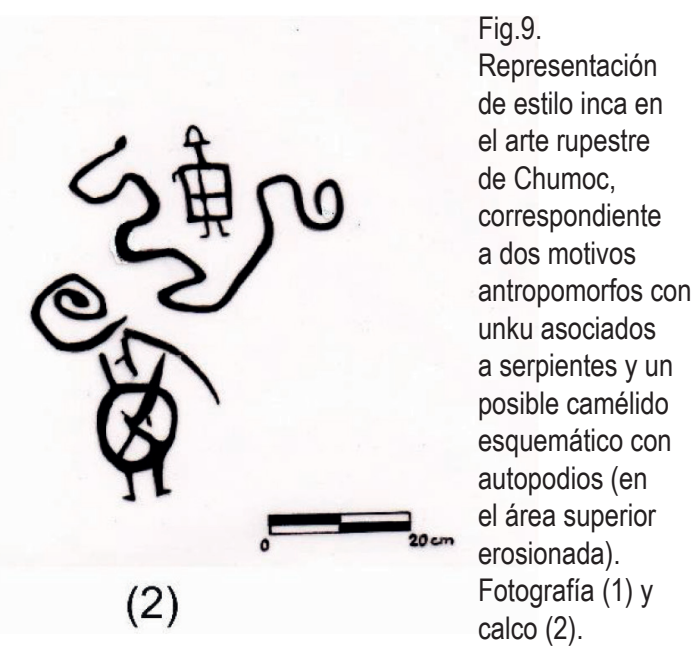

tremidades (Fig. 8), lo cual indicaría su pertenencia al estilo incaico (Véase: Berenguer 2013: 312).

Chumoc: Ubicado a medio kilómetro del pueblo de San Jerónimo, en un abrigo rocoso a 1838 m.s.n.m. Entre las representaciones zoomorfas, antropomorfas y geométricas (rojo y crema) destacamos la representación de dos motivos antropomorfos con unku representados de frente, y un posible camélido esquemático con autopodios, los cuales corresponderían a motivos de estilo incaico (Fig. 9).

Asimismo existen muchos materiales arqueológicos en poder de los comuneros de San Jerónimo, algunos de los cuales podrían ofrecernos información sobre el periodo al que pertenecieron, correlacionándolos con los sitios en los que se encontraron. Es así que el profesor Anderson Yomona Daza, nos permitió observar y fotografiar objetos que le fueron obse- 


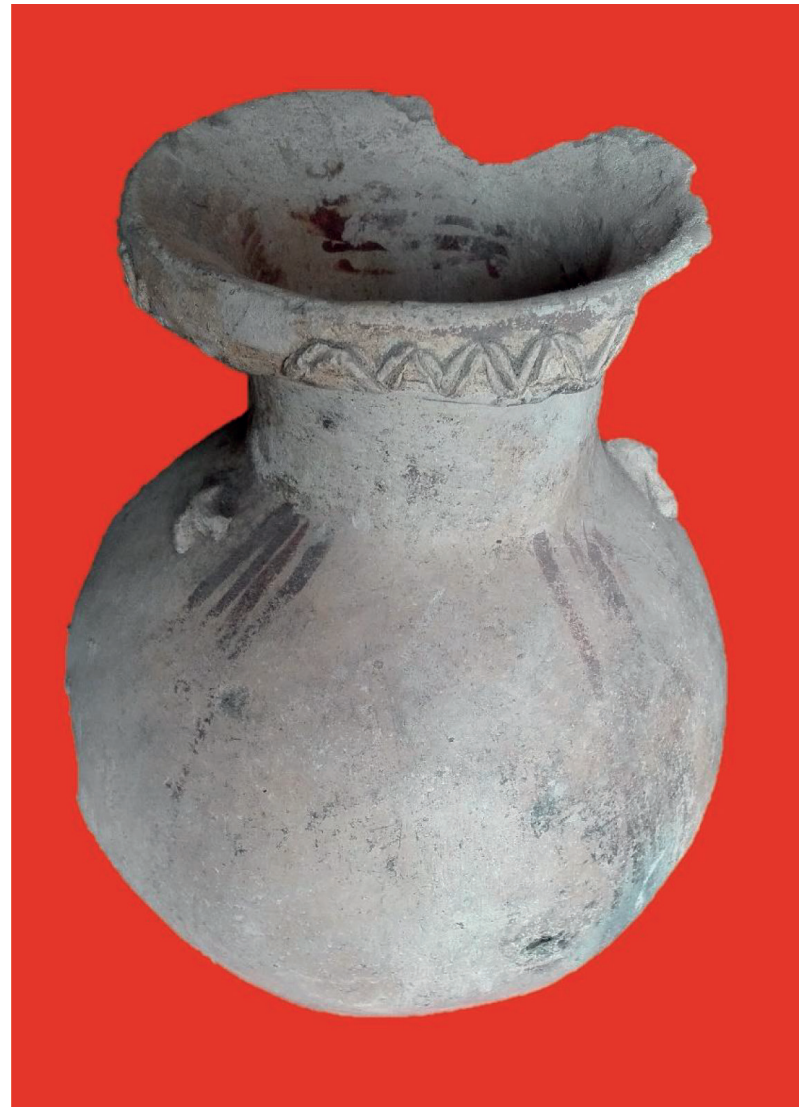

Fig.10.Ceramica de tipo Kuelap Pintado Aplicado, correspondiente a la fase Kuelap Inca (Horizonte Tardío) hallado en San Jerónimo por uno de los pobladores.

quiados por los pobladores de San Jerónimo y procederían de la misma área según le informaron estos mismos, destacando entre estos un material cerámico de tipo Kuelap Pintado Aplicado (Fig. 10), el cual es diagnóstico de la fase Kuelap Inca y por ende del periodo Horizonte Tardío, según la secuencia realizada por Arturo Ruiz Estrada (2009 [1972]: 85).

\section{Apreciaciones finales}

El asentamiento de Purumllaqta de Cuimal habría sido construido en gran parte durante el Horizonte Tardío, modificándose posteriormente durante el periodo del colonialismo español, a lo cual sumamos las transformaciones ulteriores causadas por las actividades agropecuarias, que dañaron en gran parte la planimetría del asentamiento. Durante la hegemonía inca Purumllaqta de Cuimal habría constituido una instalación importante en la administración del área abarcada por las poblaciones locales, en el territorio que ahora pertenece a la comunidad de San Jerónimo.

Asimismo, el asentamiento de Purumllaqta de Cuimal se encontraría dentro de la ruta inca del Utcubamba, la cual permitió una conexión de las tierras altas con la llanura amazónica, pertenecientes a las actuales provincias de Luya y Bagua respectivamente. Esta ruta habría sido muy importante en la conquista inca del territorio ocupado por las distintas sociedades Chachapoya y en sus expediciones hacia territorio jívaro (Astuhuaman 2009: 80), la cual a su vez permitía acceso hacia la zona del Chinchipe y por ende a territorio del actual Ecuador.

Durante el dominio inca y/o el periodo transicional (Inca-Colonial), los residentes de Purumllaqta de Cuimal y sitios aledaños habrían elaborado algunas de las representaciones rupestres en Suinte, Ayasamana y Chumoc, así como las sepulturas de algunos fardos con momias en El Tigre. Demostrando así el grado de afianzamiento incaico en el área de la actual comunidad de San Jerónimo, al implantar nuevos patrones en la arquitectura, manifestaciones rupestres y costumbres funerarias.

Como parte de la estrategia para afianzar los territorios conquistados por el imperio inca, se construyeron establecimientos con un patrón cusqueño, muchas veces con variantes locales, ya sean técnicas o morfológicas. En territorio de las sociedades "Chachapoya", al encontrarse bajo el dominio inca, se construyeron diversos asentamientos como el centro administrativo de Cochabamba (Schjellerup 2005: 211-247), Paxamarca (Davis 1988: 68; Rojas 2017: 28), Purumllaqta de Cheto (Ruiz Estrada 2004: 78) y Purumllaqta de Soloco (Crandrall 2017: 300), con el fin de controlar mejor a la población y los recursos del nuevo territorio anexado al Tahuantinsuyo.

Dentro de la organización del territorio conquistado el asentamiento de Purumllaqta de Cuimal al parecer tendría una menor jerarquía frente a los sitios mencionados anteriormente, lo cual no disminuiría su importancia en la administración del nuevo territorio anexado al Tahuantinsuyo, ya que este asentamiento se encontraba en una ruta que conectaba la llanura amazónica de Bagua con asentamientos muy importantes como Kuélap, sitio ocupado de manera extensa durante el periodo incaico (Ruiz Estrada 2009 [1972]: 15; Kauffmann, 2013: 64; Narváez, 2013: 157), y con complejos rupestres donde se plas- 
maron representaciones de estilo inca como Chanque (Ruiz Estrada 2007: 97-109), sumándose a este los sitios de Suinte, Ayasamana y Chumoc.

Una interrogante acerca de Purumllaqta de Cuimal corresponde al momento de su abandono, ya que si recurrimos a su mención en ciertas fuentes etnohistóricas del siglo XVIII (Bueno 1764: 32; de Alcedo 1786: 727) y la presencia de una inscripción en la iglesia del pueblo actual de san Jerónimo que indicaría el año de su construcción en 1861, podríamos considerar un traslado poblacional tardío, entre 1786 y 1861; sin embargo existiría la posibilidad de su abandono a finales del siglo XVI, apoyados en que muchos lugares ocupados durante el Horizonte Tardío fueron abandonados debido a las reducciones, como los casos de Purunllaqta de Soloco (Crandrall 2017: 300), Kuélap y Yaro9 (Espinoza 1967: 237). Esta y otras interrogantes podrán responderse ulteriormente, mediante investigaciones más exhaustivas.

Agradecimientos: A Miuler Villar Sánchez, quien nos informó y acompañó a la mayoría de sitios mencionados en el presente trabajo. A Ángel Ventura, José Hernández, Anderson Yomona y Reynaldo Ocampo, quienes de distintas maneras hicieron posible la presente investigación. A los arqueólogos Lic. Iván Díaz Lon, por su apoyó en la digitalización final del plano referente a Purumllaqta de Cuimal, y Dr. Arturo Ruiz Estrada por sus comentarios y recomendaciones.

\section{Referencias bibliográficas}

Arana, Petter \& Zuta, Horacio (2009). Arte rupestre en la región Amazonas. Perú.

Astuhuamán, César (2009). Incas, Jivaros y la obra de Humboldt Vues des Cordillères. HiN-Alexander von Humboldt im Netz. Internationale Zeitschrift für Humboldt-Studien. Vol. 10. No 19. 68-91. Berlín.

UGEL Bongará (2006). Bongará: Historia, leyendas y costumbres. Red Educativa Temática de Lenguaje y Comunicación. Unidad de Gestión Educativa Local de Bongará.

Bueno, Francisco (1764). Descripción de las provincias de los Obispados y Arzobispados del Virreinato del Perú. Lima: Impreso en la Oficina de la Calle de la Coca.

9 Posible nombre del sitio conocido como Ruinas del Abiseo o Gran Pajatén.
Centro Mallqui \& Espeleo Kandil (2013). Informe sobre los Yacimientos aparecidos en San Gerónimo (Provincia de Bongará, Amazonas, Perú).

Crandall, James (2017). El desarrollo espacial de las comunidades Chachapoyas bajo la dominación colonial inka y española. Boletín de Arqueología PUCP. No 23. 283-311. Lima.

Davis, Morgan (1988). Chachapoyas The Cloud People. NATI 3055. Departament of Native Studies University of Sudbury. Ontario. Canadá.

De Alcedo, Antonio (1786). Diccionario geográfico-histórico de las Indias Occidentales ó América. Tomo I. Imprenta Benito Cano. Madrid. España.

Espinoza, Waldemar (1967). Los señoríos étnicos de Chachapoyas y la alianza hispano-chacha. Revista Histórica. Tomo XXX. 224-333. Lima.

Grandez de Salinas, Francisco (1598). Títulos pertenecientes a los terrenos del pueblo de san Gerónimo.

Guillén, Sonia (2002). Las momias de la laguna de los Cóndores. Chachapoyas: el reino perdido. Integra AFP. 345-387. Lima.

Guillén, Sonia (2003). De Chinchorro a Chiribaya: los ancestros de los mallquis Chachapoya-Inca. Boletín de Arqueología PUCP No 7. 287-303. Lima.

Hostnig, Rainer (2006). Distribución, Iconografía y Funcionalidad de las Pinturas Rupestres de la Época Inca en el Departamento del Cusco, Perú. Boletín SIARB. $\mathrm{N}^{\circ}$ 20. Sociedad de Investigación del Arte Rupestre de Bolivia. 46-76. La Paz.

Hostnig, Rainer (2008). Pinturas rupestres de posible afiliación inca en el de partamento del Cusco, Perú. En Rupestreweb, http://www.rupestreweb.info/incacusco. html

Hostnig, Rainer (2017). Personajes de rango y emblemas de poder en pinturas rupestres incaicas del Valle Sagrado, Cusco, Perú. TRACCE-Online rock Art Bulletin.

Kauffmann, Federico (2013). Los Chachapoyas: orígenes y trayectoria cultural. Los Chachapoyas. Banco de Crédito del Perú, 41-79. Lima.

Kauffmann, Federico (2017). La cultura Chachapoyas. Cartolan. Lima. Perú.

Kauffmann, Federico \& Ligabue, Giancarlo (2003). Los Chachapoya (s): moradores ancestrales de los Andes amazónicos peruanos. UAP. Lima. Perú.

Morales, Daniel. (1998). Historia Arqueológica del Perú (del paleo-lítico al imperio Inca). Compendio Histórico del Perú. Editorial Milla Batres. Lima. Perú. 
Narváez, Alfredo (2013). Kuélap: Centro del poder político religioso de los Chahapoyas. Los Chachapoyas. Banco de Crédito del Perú. 87-159. Lima.

Quintana, Santiago (2011). Descubren sarcófagos en San Jerónimo, Bongará, Amazonas. En: Cultura VivaAmazonas [Blog]. https://culturavivaenamazonas. blogspot.com/2011/09/descubren-sarcofagos-en-sanjeronimo.html

Quintana, Santiago (2013). Encuentran más sarcófagos en San Jerónino, Bongará. En: Cultura Viva-Amazonas [Blog]. https://culturavivaenamazonas.blogspot. com/2013/07/encuentran-mas-sarcofagos-en-san.html

Rojas, Manuel (2017). Paxamarca: Un asentamiento Chacha-Inca, en la Región Amazonas. XXV Congreso Nacional de Estudiantes de Arqueología. Universidad Nacional De Trujillo. 27-28. Trujillo.

Ruiz, Marta (2002). Unkus, caminos y encuentros. Revista Andina No 34. 199-215. Cusco.

Ruiz, Marta \& Chorolque, Domingo (2012). Arte rupestre y la presencia inka en el Pukara de Rinconada, puna de Jujuy, Argentina. Investigaciones Sociales. Vol. 16. No 28. UNMSM. 343-349. Lima.

Ruiz Estrada, Arturo (2004). Purumllacta: Un centro administrativo incaico en Chachapoyas. Investigaciones Sociales. Vol. 8. No 13. UNMSM. 73-84. Lima.

Ruiz Estrada, Arturo (2007). Chanque: Un Santuario de Arte Rupestre en la Región de Amazonas. Actas del Primer Simposio Nacional de Arte Rupestre. Cusco. Actas y Memorias del Instituto Francés de Estudios Andinos. Tomo 12. Lima.

Ruiz Estrada, Arturo (2009 [1972]). La alfarería de Kuelap: tradición y cambio. Serie Tesis. No 4. Avqi Ediciones. Lima.
SCHJEllerup, Inge (2005). Incas y españoles en la conquista de los Chachapoya. Fondo PUCP e IFEA. Lima. Perú.

SepúlvedA, Marcela (2004). Esquemas visuales y emplazamiento de las representaciones rupestres de camélidos del Loa Superior en tiempos incaicos. ¿Una nueva estrategia de incorporación de este territorio al Tawantinsuyu? Chungara, Revista de Antropología Chilena. Volumen 36, No 2. 439-451. Arica.

TAYLOR, Gerald (1996). La tradición oral quechua de Chachapoyas. Instituto Francés de Estudios Andinos \& Archivo de la Tradición Oral Quechua. Lima. Perú.

Torres, Carlos (1986). Boletín Kuelap. No 68. Instituto Nacional de Cultura - Amazonas. Chachapoyas.

Torres, Carlos (1994). Boletín Kuelap. No 105. Instituto Nacional de Cultura - Amazonas. Chachapoyas.

Vetter, Luisa (2008). Plateros indígenas en el virreinato del Perú: siglos XVI y XVII. Universidad Nacional Mayor de San Marcos y Compañía de Minas Buenaventura. Lima. Perú.

VILlar, Miuler (2012). Descubrimiento de un yacimiento de sarcófagos. En: Legado ancestral [Blog]. http://legadoancestral.blogspot.pe/2012/01/descubrimiento-deun-yacimientode.html

Villar, Miuler (2013). Nuevo descubrimiento de sarcófagos en San Jerónimo. En: Legado ancestral [Blog]. http://legadoancestral.blogspot.pe/2013/07/nuevodescubrimiento-desarcofagos-en.html

VILlar, Miuler (2013). Tercer descubrimiento de sarcófagos en San Jerónimo, amazonas. En: Legado ancestral [Blog]. http://legadoancestral.blogspot.pe/2013/11/ tercer-descubrimiento-desarcofagos-en.html 